\title{
Interaction of Candida krusei with human neutrophils in vitro
}

\author{
M. D RICHARDSON and F. DONALDSON
}

Regional Mycology Reference Laboratory, Department of Dermatology, University of Glasgow, Robertson Building, 56 Dumbarton Road, Glasgow G11 6NU

\begin{abstract}
Summary. In human neutrophil monolayer assays the percentage phagocytosis of Candida krusei by neutrophils was found to be significantly lower $(9 \%)$ than that for $C$. albicans $(37 \%)$. Both organisms required opsonisation with complement products for ingestion. The number of competent neutrophils phagocytosing $C$. krusei was increased with: antisera specific to $C$. albicans $(49 \%)$; the neutrophil chemo-attractant formyl-methionyl-leucylphenylalanine (fmlp) $(49 \%)$; mannan extracted from the cell wall of C. albicans $(72 \%)$; and a crude cell extract from $C$. krusei $(61 \%)$. In the case of $C$. albicans all but one of these methods increased the proportion of phagocytosing neutrophils per slide. The data provide evidence for differences in quantitative phagocytosis of $C$. krusei and C. albicans and suggest that $C$. krusei is resistant to phagocytosis in vitro.
\end{abstract}

\section{Introduction}

Candida krusei is emerging as a common commensal of the mouth, throat and urinary tract. ${ }^{1}$ It is also an emerging pathogen amongst immunologically incompetent hosts. ${ }^{1}$ It is seen in AIDS, cancer treatment, steroid use, drug abuse and primary immunodeficiencies. All involve neutropenia which can result in widespread candidosis.

The emergence of $C$. krusei as a pathogen in both competent and compromised hosts has increased over the past three decades. ${ }^{1}$ This could be due to the increase in the population of immunocompromised hosts, which has allowed the development of a reservoir of $C$. krusei, in conjunction with the resistance of $C$. krusei to antifungal agents. Since the newer azole antifungal agents, fluconazole and itraconazole, are currently used for prophylaxis in cancer patients, it is possible that $C$. krusei superinfections may emerge.

Protection against systemic candida infections is mainly attributed to neutrophils. Although a major role for neutrophils in normal (non-specific) host defence against $C$. albicans has been firmly established, little is known about the interaction between $C$. krusei and neutrophils. Neutrophils undoubtedly play a vital role in host defence against $C$. krusei. The shorter survival and higher fungal burden noted in immunosuppressed animals suggest a potential role for neutrophils and macrophages as defence mechanisms

Received 9 June 1994; accepted 29 June 1994. for disseminated C. krusei infection. ${ }^{2}$ This implicates any factor which could potentially modulate phagocytosis of $C$. krusei by neutrophils in vivo. Based on previous studies, ${ }^{3,4}$ there appears to be some correlation between the capacity of Candida spp. to oppose phagocytic killing in vitro and virulence in experimental models but the difference between pathogenic and non-pathogenic species becomes significant only when the rate of killing and the spectrum of active effectors are considered. Several studies have shown that the cell wall of $C$. krusei differs from that of $C$. albicans. For example, the glucan content of $C$. krusei increases on administration of a bis-triazole and the expression of an $\mathrm{iC} 3 \mathrm{~b}$ receptor is less than that found on C. albicans. ${ }^{5}$ These differences suggest differences in their interaction with host cells.

\section{Materials and methods}

\section{Organisms}

Six clinical isolates of $C$. krusei were obtained from the National Collection of Pathogenic Fungi, PHLS Mycology Reference Laboratory, Bristol. C. albicans and Saccharomyces cerevisiae were obtained from the Regional Mycology Reference Laboratory, Glasgow. Subcultures and further growth of the organisms was on glucose peptone chloramphenicol agar (GPC) at $37^{\circ} \mathrm{C}$.

All cultures were 3-4 days old when used in the neutrophil assays. Surface growth of yeast cells was 
emulsified in $10 \mathrm{ml}$ of distilled water to give a single cell suspension of blastoconidia. The cell suspension was then diluted to $1 \times 10^{5}$ cells $/ \mathrm{ml}$ in Hanks's Balanced Salts Solution (HBSS).

\section{Opsonin}

Unpurified isologous serum complement as a component of peripheral blood was used as an opsonin where indicated. Serum was stored at $-70^{\circ} \mathrm{C}$.

\section{Heat inactivation of C. krusei blastoconidia}

Yeast cells were emulsified in $10 \mathrm{ml}$ of distilled water, and then held in a boiling water bath for $20 \mathrm{~min}$.

\section{Antisera}

Serum from a histopathologically proven case of systemic candidosis was used as a source of specific anti-C. albicans immunoglobulin. Inactivation of complement was achieved by heating the serum at $56^{\circ} \mathrm{C}$ for $30 \mathrm{~min}$.

\section{$N$-formylmethionyl-leucylphenylalanine ( $f m l p)$}

Fmlp (Sigma) was diluted $10^{-2} \mathrm{M}$ in DMSO and stored at $-70^{\circ} \mathrm{C}$. This stock solution was then diluted in HBSS to the desired concentration for each set of experimental conditions.

\section{Mannan}

Purified cell-wall mannan of $C$. albicans was obtained as a component of the Pastorex Candida latex agglutination kit (Diagnostics Pasteur, Marnesla-Coquette, France).

\section{Cell-wall extract}

A heavy suspension $\left(10^{9}\right.$ cells $\left./ \mathrm{ml}\right)$ of blastoconidia of C. krusei in $10 \mathrm{ml}$ of distilled water was sonicated for $30 \mathrm{~min}$ in a water bath. The cell suspension was assessed microscopically to determine the degree of cell lysis. The supernate from centrifugation of the suspension constituted a crude extract of cell-surface antigen.

\section{Preparation of neutrophil monolayers}

Monolayers of glass-adherent neutrophils were prepared as described previously. ${ }^{6}$ Briefly, drops of blood from finger-tip punctures (single donor throughout) were placed on $16-\mathrm{mm}$ diameter coverglasses. The blood drops were incubated for $30 \mathrm{~min}$ at $37^{\circ} \mathrm{C}$ in a humidified chamber. They were then immersed in saline $0.85 \% \mathrm{w} / \mathrm{v}\left(37^{\circ} \mathrm{C}\right)$ and agitated to remove the adherent red cells. The coverglasses were then washed again in saline, resulting in confluent neutrophil monolayers.

\section{Assessment of phagocytosis}

Neutrophil monolayers were placed in the wells of "Repli" (Sterilin) tissue culture plates containing $1 \mathrm{ml}$ of a $1 \times 10^{5} \mathrm{ml}$ conidial suspension. Normal human serum $(100 \mu \mathrm{l})$ was added to each monolayer immediately before incubation at $37^{\circ} \mathrm{C}$ for $30 \mathrm{~min}$. The monolayers were then washed twice in saline $0.85 \%$ and inverted over glass cavity slides. Monolayers were examined within $5 \mathrm{~min}$ by high power $(\times 100$ objective) oil immersion microscopy. Ten randomly selected fields of view were examined; the only constraint was that at least two neutrophils be present in each field. The total number of neutrophils/field was counted and the number of conidia ingested by each neutrophil was recorded. From these observations, the following parameters were calculated: percentage phagocytosis (number of neutrophils with one or more ingested conidia/total neutrophil number), and phagocytic index (number of ingested conidia/ neutrophil number containing one or more conidia).

\section{Pre-treatment of neutrophils}

Before the addition of conidia, neutrophil monolayers were immersed in either HBSS alone, or fmlp and HBSS, and incubated for $20 \mathrm{~min}$ at $37^{\circ} \mathrm{C}$. Monolayers were then washed to remove excess fmlp before transfer to conidial suspensions. Pre-treatment with mannan was performed in a similar manner.

\section{Statistical analysis}

All data were analysed by Student's $t$ test. As replicate experiments were performed on different days and at different times of day, the test results were compared against the control values obtained at the same time. The pooled variance and statistical significance for all control and test values were also calculated to overcome the problem of day to day variability.

\section{Results}

\section{Phagocytosis}

The percentage phagocytosis of C. krusei blastoconidia was compared to that of $C$. albicans and S. cerevisiae. C. krusei was ingested by 7 SD $1.9 \%$ of the neutrophils observed. This was in contrast to $C$. albicans (37 SD 5.1; p $<0.001)$ and $S$. cerevisiae $(28$ SD $6.3 \% ; \mathrm{p}<0.001)$ ). Ingestion of $C$. krusei did not approach that of $C$. albicans after $90 \mathrm{~min}$. Maximal phagocytosis was seen after $60 \mathrm{~min}$, but this was not significantly different from the phagocytosis seen at $30 \mathrm{~min}$. The ability of C. krusei to evade ingestion was not dependent on metabolic activity. Heat-killed blastoconidia were not phagocytosed significantly compared to viable cells ( $p>0 \cdot 1)$ (fig. 1). Phagocytosis was not strain dependent. All six strains were able to 


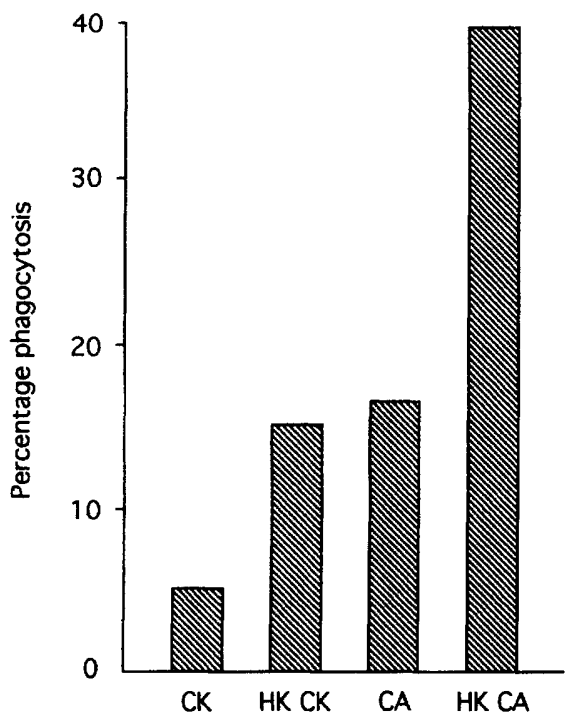

Fig. 1. Phagocytosis of viable and heat-killed blastoconidia of $C$. krusei and $C$. albicans by human neutrophils: CK, C. krusei; HK CK, heat-killed C. krusei; CA, C. albicans; HK CA, heat-killed C. albicans.

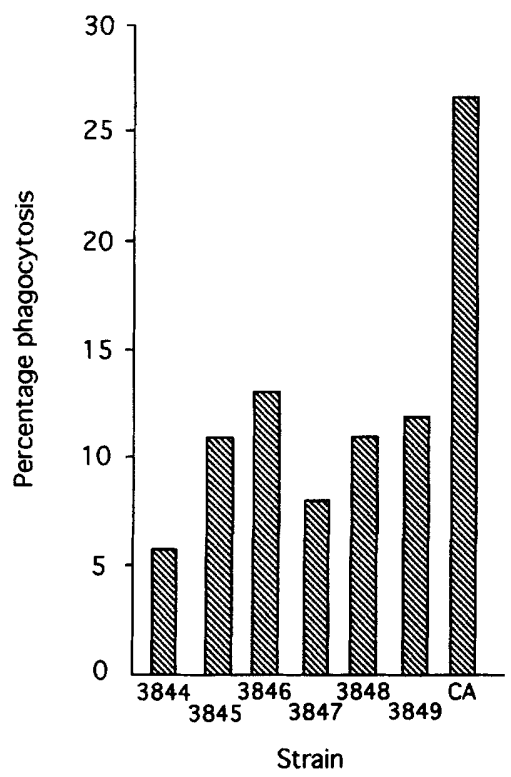

Fig. 2. Phagocytosis of blastoconidia of $C$. krusei isolates (NCPF 3844-3849) and C. albicans (CA).

resist ingestion ( $p>0 \cdot 1)$ (fig. 2). Ingestion of all strains of C. krusei was significantly lower than that seen for C. albicans $(\mathrm{p}<0.001)$.

Upregulation of phagocytosis by specific anti-C. albicans immunoglobulin

When anti-C. albicans immunoglobulin was added to the neutrophil assay in addition to serum complement, there was a significant increase in ingestion of C. krusei blastoconidia $(\mathrm{p}<0.001)$. Phagocytosis in the presence of complement-inactivated specific antiserum was not significantly different from that seen with antiserum containing complement.

Maximal ingestion was seen when antiserum and serum complement were added contemporaneously to blastoconidia in the neutrophil assay. There was no

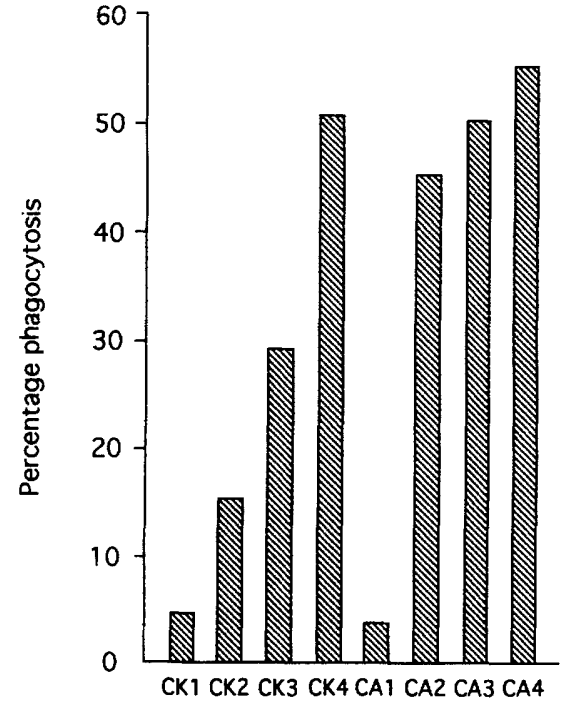

Fig. 3. Phagocytosis of blastoconidia of $C$. krusei and C. albicans in the presence of serum complement and anti-C. albicans immunoglobulin: CK1, C. krusei, complement not present; CK2, C. krusei, complement present; CK3, C. krusei, anti-C. albicans immunoglobulin present; CK4, $C$. krusei, complement and anti- $C$. albicans immunoglobulin present; CA1-CA4, C. albicans, as for $C$. krusei.

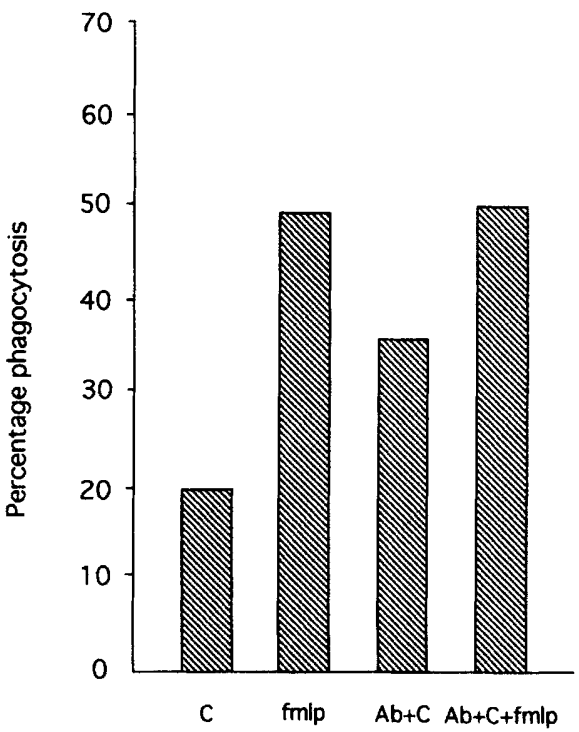

Fig. 4. Effect of fmlp in the presence of serum complement and antiC. albicans immunoglobulin on phagocytosis of C. krusei blastoconidia: C, complement; fmlp, $\mathrm{N}$-formylmethionylleucylphenylalanine; $\mathrm{Ab}$, anti-C. albicans immunoglobulin.

significant difference between $C$. krusei and C. albicans (fig. 3). The antiserum was tested for cross reactivity between the two Candida strains by running absorbed sera after the addition of blastoconidia for $3 \mathrm{~h}$ on counterimmuno-electrophoresis gels. Antibodies specific to $C$. albicans were present as they were absorbed by $C$. albicans blastoconidia. Blastoconidia of $C$. krusei did not remove anti-C. albicans immunoglobulin.

\section{Activation of neutrophils with fmlp}

Pre-exposing neutrophil monolayers to $\mathrm{fmlp}$ increased ingestion of $C$. krusei from 21 to $49 \%$. The level of ingestion in the presence of complement and anti-C. albicans immunoglobulin was $48 \%$ (fig. 4). However, differences between complement + 


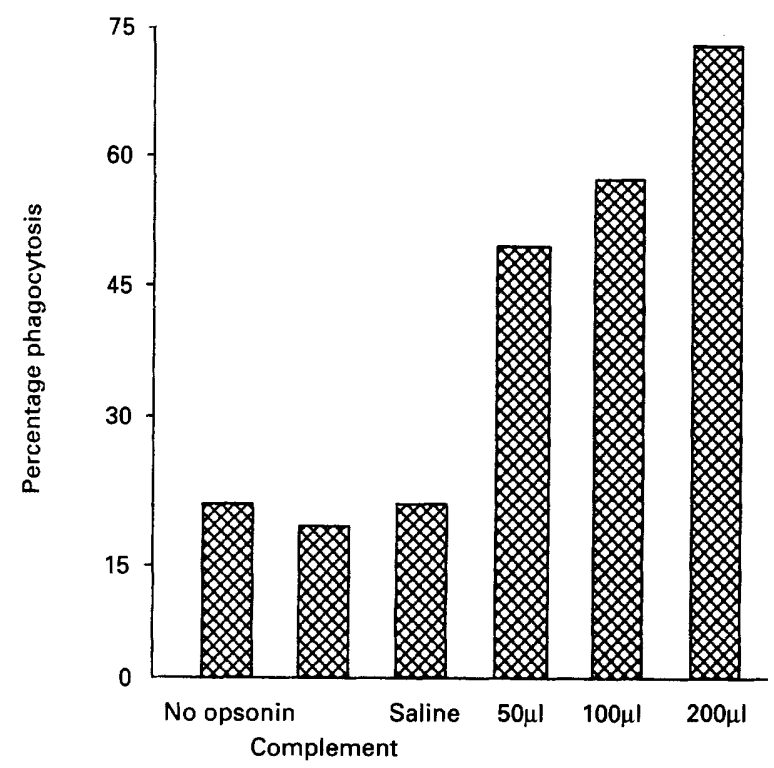

Fig. 5. Phagocytosis of C. krusei blastoconidia by human neutrophils in the presence of $C$. albicans mannan $(50-200 \mu \mathrm{l})$.

fmlp + anti-C. albicans immunoglobulin, and complement + fmlp were not significantly different.

\section{Mannan as a stimulator of phagocytosis}

Phagocytosis of C. krusei was increased significantly when soluble mannan was added at the same time in the neutrophil assay. This was a dose-dependent phenomenon (fig. 5). Pre-exposure of neutrophils to mannan had no significant effect on ingestion $(\mathrm{p}>0 \cdot 1)$.

\section{Cell-surface extract of C. krusei as a stimulator of ingestion}

An increase in ingestion of $C$. krusei blastoconidia from 22 to $43 \%$ was seen when the crude cell extract was added to the assay $(p<0.001)$. The extract alone did not replace the activity of serum complement $(\mathrm{p}>$ $0 \cdot 1)$ but when extract and complement were added contemporaneously, ingestion was potentiated $(\mathrm{p}<$ $0 \cdot 001)$.

\section{Discussion}

C. krusei is an uncommon but increasingly recognised cause of disseminated fungal infection in immunocompromised patients. ${ }^{1}$ The host defence mechanisms against the organism are not well understood.

Differences in size and cell-wall architecture suggested that there may be differences between $C$. albicans and C. krusei in their interaction with human neutrophils. The typical morphology of $C$. krusei is predominantly cylindrical compared with the considerably smaller spherical or oval shape of C.albicans. The size of most blastoconidia of $C$. krusei is (3-5) $\times(6-20) \mu \mathrm{m}$. Phagocytosis of all strains examined was lower than that seen for C. albicans or $S$. cerevisiae. However, the inability of neutrophils to ingest $C$. krusei was not entirely dependent on cell size or morphology, as many of the yeast cells in the Saccharomyces cell population were larger than $C$. krusei cells.

Fungal surface carbohydrates have been implicated most often as recognition sites for phagocytes. ${ }^{7}$ However, the indirect nature of the evidence which is based on competitive inhibition of phagocytosis by defined molecules dictates caution in the interpretation of results. The possibility of cell-surface antigens of $C$. krusei conferring protection against phagocytosis was investigated by heat inactivating blastoconidia before exposure to neutrophils. The low level of ingestion was not enhanced. It is apparent that $C$. krusei does not have a level of metabolic activity which hinders binding to neutrophil surfaces or phagocytosis. It is likely that most of the polysaccharide moiety on the cell surface was destroyed by this process, but it is evident that the cell wall was not made more antigenic through heat inactivation. Human monocyte receptors for B-glucan have been linked to phagocytosis of heat killed blastoconidia, perhaps recognising a heptoglucoside thought to mediate zymosan particle ingestion. ${ }^{7}$ However, monocytes may not utilise identical receptors in the recognition of live and boiled or fixed C. albicans blastoconidia. Others suggest the involvement of macrophage mannose receptors in the phagocytosis of C. albicans, but the purity of inhibitory "mannans" is not well documented in many studies. ${ }^{7}$ Mannan is bound to neutrophils by mannan-binding proteins. ${ }^{8}$ This binding has been shown to upregulate phagocytosis but not intracellular killing by neutrophils. ${ }^{8}$ In the present study, mannan was not active if it was preincubated with neutrophils, suggesting that any effect contributed by this sugar was in collaboration with serum complement. Furthermore, all monoclonal antibodies devised so far against a $C$. albicans mannan are not cross-reactive with $C$. $k r u s e i^{5,9-11}$

Specific effects of opsonins on phagocytosis and triggering of subsequent responses depend on the particular fungal and phagocytic cell type. Potential opsonins may or may not be deposited on cell walls in configurations or at sites that facilitate recognition by phagocyte receptors. Opsonin deposition may supplement or mask fungal loci that stimulate phagocytosis, sometimes changing the receptors that initiate phagocyte activation.

The presence of specific anti- $C$. albicans immunoglobulin enhanced ingestion of $C$. krusei two-fold. This did not appear to be due to an increased level of complement in the assay, but was dependent on complement being present. However, specific antibody did not appear to bind to $C$. krusei, as shown by the absorption studies. Nevertheless, the increase in ingestion would suggest that there was some crossreactivity between the cell surface antigens of $C$. albicans and $C$. krusei. Alternatively, the affinity of anti-C. albicans serum for $C$. krusei was only sufficient to activate the complement cascade thereby 
stimulating more neutrophils but not increasing the number of internalised yeast cells per neutrophil.

Various host factors can modulate phagocyte responses. Neutrophil activity against a broad array of fungal pathogens can be augmented by cytokines and chemo-attractants. The bacterial chemo-attractant fmlp is very effective in activating neutrophils. In neutrophil monolayer assays fmlp has been shown to enhance phagocytosis of Aspergillus fumigatus ${ }^{12}$ and C. albican $s^{13}$ by human neutrophils. For C. krusei the evidence is just as clear; fmlp increased the percentage phagocytosis to levels similar to $C$. albicans. The synergy between fmlp and anti- $C$. albicans immunoglobulin suggests that complement was activated even further.

The mechanism of inhibition of phagocytosis of $C$. krusei is not known and the specific mechanism by which $C$. krusei evades ingestion has not been established. Several hypotheses can be put forward. Firstly, C. krusei may be a poor activator of complement. In the presence of complement alone $C$. krusei was not susceptible to phagocytosis. This contention is

\section{References}

1. Samaranayake H, Samaranayake LP. Candida krusei: biology, epidemiology, pathogenicity and clinical manifestations of an emerging pathogen. $J$ Med Microbiol 1994; 41 : 295-310.

2. Anaissie E, Haychem R, K-Tin-U C, Stevens LC, Bodey GP. Experimental haematogenous candidiasis caused by Candida krusei and Candida albicans: species differences in pathogenicity. Infect Immun 1993; 61: 1268-1271.

3. Borg-von-Zeplin M, Schuff-Werner P. Chemiluminescence of polymorphonuclear granulocytes in the presence of selected Candida species. Mycoses 1992; 35: 121-129.

4. Vecchiarelli A, Bistoni F, Cenci E, Perito S, Cassone A. In-vitro killing of Candida species by murine immunoeffectors and its relationship to the experimental pathogenicity. Sabouraudia $1985 ; 23$ : 377-387.

5. Cassone A, Torosantucci A, Boccanera M, Pellegrini G, Palma C, Malavasi F. Production and characterisation of a monoclonal antibody to a cell-surface, glucomannoprotein constituent of Candida albicans and other pathogenic Candida species. J Med Microbiol 1988; 27: 233-238.

6. Richardson MD, Smith $H$. Resistance of virulent and attenuated strains of Candida albicans to intracellular killing by human and mouse phagocytes. J Infect Dis 1981 ; 144: $557-564$.

7. Diamond RD. The fungal cell wall and vertebrate phagocytosis. In: Latgé J-P, Boucias D (eds) Fungal cell wall and immune response. NATO ASI Series H, vol 53. Berlin, Springer-Verlag. 1991: 331-340.

8. Palma C, Serbousek D, Torosantucci A, Cassone A, Djeu JY. supported by the finding that $C$. krusei has few iC $3 \mathrm{~b}$ receptors on its surface. ${ }^{14}$ When heat-killed blastoconidia were not phagocytosed more readily it may have been that neutrophils recognised the organisms as dead and, therefore, phagocytosis did not take place and it may be that defensins which are used in intracellular killing are only directed against live cells. Another mechanism by which C. krusei could evade phagocytosis is through a mannan structure which is not specific for mannan binding proteins (or other neutrophil surface molecules).

These results suggest that neutrophils alone may not efficiently control $C$. krusei, since phagocytosis is significantly less than that which is seen with $C$. albicans. The low level of binding to neutrophils and minimal ingestion are similar to those seen with Trichosporon beigelli. ${ }^{15} \mathrm{We}$ are currently examining cross-reactivity between $C$. krusei and T. beigelli in our neutrophil monolayer model.

We thank the technical staff of the Regional Mycology Reference Laboratory for their support services and Dr I. C. McKay for his statistical expertise.

Identification of a mannoprotein fraction from Candida albicans that enhances human polymorphonuclear leukocyte (PMNL) functions and stimulates lactoferrin in PMNL inhibition of candidal growth. J Infect Dis 1992; 166: 1103-1112.

9. Pauliak V, Sandula J. Cross-reactivity of pathogenic Candida mannans studies by enzyme-linked immunosorbent assay (ELISA) and precipitin methods. Mycoses 1988; 31: 34-39.

10. Georges E, Garrigues ML, Stynen D, Poirot JL. In-vitro specificity of an anti-mannan monoclonal antibody (Mab) and evaluation of a mannan Mab-sensitized latex in experimental disseminated candidosis. J Mycol Med 1991; 1: $21-24$.

11. Nnalue NA, Weintraub A, Lindberg AA. Properties of a rat monoclonal antibody reactive with both the mannan of Candida species and the $\mathrm{O}$-antigen 6,7 polysaccharide of serotype $C_{1}$ salmonellae. Infect Immun 1991; 59: 229-233.

12. Richardson MD, Patel M. Stimulation of neutrophil phagocytosis of Aspergillus fumigatus by interleukin-8 and Nformylmethionyl-leucylphenylalanine. $J \mathrm{Med}$ Vet $\mathrm{Ml}$ 'col 1994; (in press).

13. Djeu JY, Matsushima K, Oppenheim JJ, Shiotsuki K, Blanchard DK. Functional activation of human neutrophils by recombinant monocyte-derived neutrophil chemotactic factor IL-8. J Immunol 1990; 144: 2205-2210.

14. Hostetter MK. Function of integrin analogues in adhesion. $J$ Mycol Med 1992; 2: 14-18.

15. Lyman CA, Walsh TJ. Phagocytosis of medically important yeasts by polymorphonuclear leukocytes. Infect Immun 1994; 62 : 1489-1493. 